\title{
Study on Extraction Technology of Pitaya Polysaccharide
}

\author{
Feng Xuehua*, Song Zurong, Tao Ali, Gong Panpan \\ College of Pharmacy, Anhui Xinhua University, Anhui, Hefei, 230088
}

\begin{abstract}
Using pitaya as raw material, this paper used ethanol subsiding method to study the extraction technology of pitaya polysaccharide by single factor test and orthogonal test. Through orthogonal test, we have found the optimum extraction conditions of pitaya polysaccharide as follows: extraction temperature $60^{\circ} \mathrm{C}$, solid-liquid ratio $1: 30$, extraction time $3 \mathrm{~h}$. Under the optimal extraction conditions, the yield of pitaya polysaccharide was $1.13 \%$.
\end{abstract}

\section{Introduction}

Hylocereusundulatus Britt, also known as Pitaya fruit, Jade Dragon Fruit, Red Dragon Fruit and Dragon Pearl Fruit, is a fruit cultivar of Trigonocarpus genus in Cactaceae. It is a kind of cactaceous plant that can blossom and bear fruit, and its fruit is edible. It is native to Costa Rica, Cuba, Mexico and other tropical areas in Central America. After being introduced into China, it is mainly distributed in the southern provinces, such as Guangdong, Hainan, Fujian, Taiwan and so on.

Pitaya is a kind of perennial climbing succulent. The rootstock is dark green, very stout and triangular-shaped. It has a wavy edge and clings to other plants. The flower is white and the huge ovary is inferior, which has the name of "the king flower". The fruit is olive like, the outer surface has the fleshy scale outwardly curls, and the flesh is covered with black small seeds. The texture is mild and the taste is fragrant.

The stem of pitaya is rich in plant polysaccharides, accounting for about $7 \%$ of the dried product ${ }^{[2]}$. According to the results of related scientific experiments, plant polysaccharides have high bioactivity and can maintain the biological function of organisms. This paper studied the extraction technology of pitaya polysaccharide using pitaya as raw material. The ethanol subsiding method was used to extract polysaccharide in pitaya. The optimal extraction conditions were found through single factor experiment and orthogonal test, and the content of polysaccharide was determined by phenol-sulfuric acid method [3], which provided certain theoretical basis for the further processing of pitaya fruit and the further research or development in related fields.

\section{Ingredients and Methods}

\subsection{Experimental Materials}

Ingredients: Hainan pitaya; Reagents: petroleum ether, 95\% ethanol, anhydrous ethyl ether, glucose, chloroform, anhydrous ethanol, n-butanol, phenol, acetone, sulfuric acid and other reagents are analytical pure.

FA-244 electronic balance; digital display constant temperature water bath; rotary evaporator; UV1000 visible spectrophotometer; centrifuge; thermostatic oven.

\subsection{Experimental Methods}

\subsubsection{Material Treatment}

Wash, peel, slice, dry and grind fresh pitaya at $50^{\circ} \mathrm{C}$ and store it in reagent bottles for later use.

\subsubsection{Extraction Process}

Pitaya
$\downarrow$
Petroleum ether degreasing
$\downarrow$
Hot water extraction $\left(90^{\circ} \mathrm{C}\right)$
$\downarrow$

After centrifugation, taking the supernatant (4000r/min,

$$
\begin{gathered}
30 \mathrm{~min}) \\
\downarrow \\
\text { Decompressing concentration } \\
\downarrow \\
\text { 95\% ethanol precipitation washing } \\
\downarrow \\
\text { Vacuum dried coarse products } \\
\downarrow \\
\text { Purification of crude polysaccharides }
\end{gathered}
$$

\subsubsection{Experimental Procedures}

\subsubsection{Preparation of Crude Polysaccharides}

Sample treatment: peel, slice, dry and grind the pitaya, and store the sample in a dry reagent bottle; 
Degreasing: weigh $30 \mathrm{~g}$ pitaya powder, add $100 \mathrm{~mL}$ ether to reflux for $1 \mathrm{~h}$, extract and filter, and air dry ${ }^{[4]}$;

Hot water extraction: after the degreasing operation is completed, weigh $3 \mathrm{~g}$ pitaya powder, add water in different proportions, and undertake extraction at different temperatures;

Centrifugation: centrifuge at $4000 \mathrm{r} / \mathrm{min}$ for $30 \mathrm{~min}$, and take the supernatant;

Decompressing concentration ${ }^{[5]}$ : after centrifugation, decompresse and concentrate the polysaccharide solution;

Ethanol precipitation: after the completion of the concentration step, add a certain amount of $95 \%$ ethanol to the solution, stir it while adding, place it overnight, then filter it the next day, and wash it for several times with anhydrous ethanol, acetone and anhydrous ether respectively ${ }^{[6]}$;

Vacuum drying: after filtering and washing the material, then start vacuum freeze drying, so as to get the pitaya polysaccharide crude products.

\subsubsection{Purification of crude polysaccharides -- Sevage method}

Take the advantage of the denaturation of proteins in organic solvents such as trichloroethane, and mix the crude polysaccharide extract with Sevage reagent (5:1). Then shake, stir it for $20 \mathrm{~min}$, and put it into a centrifuge at $4000 \mathrm{r} / \mathrm{min}$ for $20 \mathrm{~min}$. The denatured protein is located at the junction of the extract and Sevage reagent. Remove the denatured protein and repeat the steps several times until there is no turbidity precipitation in the middle ${ }^{[7]}$.

\subsubsection{Determination of Polysaccharide Content}

\subsubsection{Preparation of Solution}

Preparation of glucose standard solution: accurately weigh $60 \mathrm{mg}$ of dried glucose, put it in a $100 \mathrm{~mL}$ volumetric flask, dilute it with water to the scale, shake it well, and then get the glucose standard solution.

Preparation of $4 \%$ phenol: take a precise measure of $4 \mathrm{~mL}$ phenol, put it into a $100 \mathrm{~mL}$ volumetric flask, dilute it with water to the scale, shake it evenly, and get it.

\subsubsection{Production of Standard Curve}

Take a precise measure of glucose standard solution, respectively $0.0 \mathrm{~mL}, 1.0 \mathrm{~mL}, 1.5 \mathrm{~mL}, 2.0 \mathrm{~mL}, 2.5 \mathrm{~mL}$ and $3.0 \mathrm{~mL}$ in the $50 \mathrm{~mL}$ volumetric flasks, add water dilute to scale, and shake them well. Take $2 \mathrm{~mL}$ of each diluted solution and place them in tubes with plug, label group No. $1-6$, add $1 \mathrm{~mL}$ of $4 \%$ phenol solution respectively, and shake them well. Then add $7.0 \mathrm{~mL}$ concentrated $\mathrm{H} 2 \mathrm{SO} 4$ immediately, shake them while adding, heat them in water bath at $40^{\circ} \mathrm{C}$ for 30 minutes, then place them in ice water bath for 5 minutes. Measure the absorbance of each group at $490 \mathrm{~nm}$, and the experimental data are shown in Table 1.

Table 1 Measured values of standard curve

\begin{tabular}{ccccccc}
\hline & 1 & 2 & 3 & 4 & 5 & 6 \\
\hline $\mathrm{C}\left(\mathrm{ug} \cdot \mathrm{mL}^{-1}\right)$ & 0 & 2.4 & 3.6 & 4.8 & 6.0 & 7.2 \\
Absorbance $/(\mathrm{g} \cdot \mathrm{cm})$ & 0 & 0.136 & 0.202 & 0.257 & 0.323 & 0.384 \\
\hline
\end{tabular}

The solution concentration is taken as the abaxial axis, the unit is ug $\cdot \mathrm{mL}^{-1}$, the absorbance is taken as the vertical axis, and the unit is $\mathrm{L} /(\mathrm{g} \cdot \mathrm{cm})$. Draw the standard curve, then obtain the linear regression equation as follows: $\mathrm{Y}=0.053 \mathrm{x}$ $+0.0049, \mathrm{R}^{2}=0.999$. The results are shown in Figure 1 .

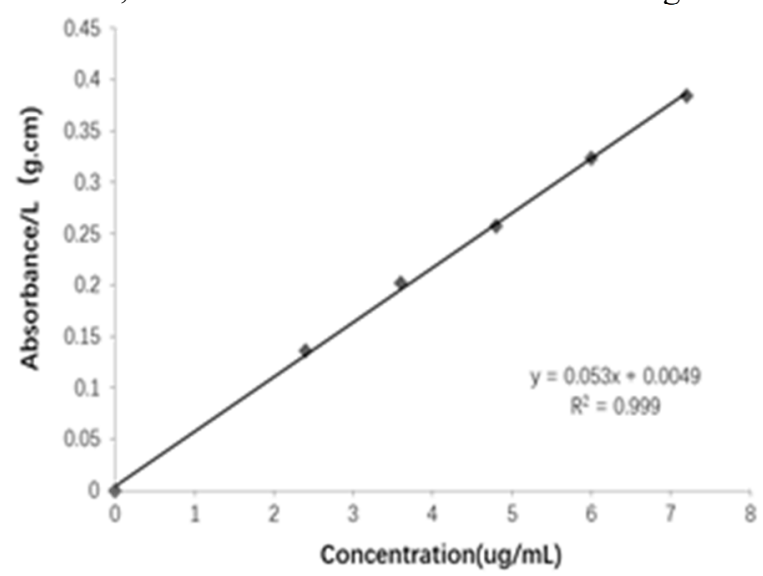

Fig. 1 Standard curve of glucose

\subsubsection{Determination of Polysaccharide Content of the Sample}

Dissolve and dilute the extracted polysaccharide into a $100 \mathrm{~mL}$ volumetric flask. Precisely take $2 \mathrm{~mL}$ diluted and constant volume into a $50 \mathrm{~mL}$ volumetric flask as sample solution. Take a precise measure of sample solutions of $0.0 \mathrm{~mL}$ and $2.0 \mathrm{~mL}$, place them in $10 \mathrm{~mL}$ tubes with plug respectively. Then add $1 \mathrm{~mL}$ of $4 \%$ phenol solution respectively, shake them well, and add $7.0 \mathrm{~mL}$ concentrated H2SO4 immediately. Shake them while adding, heat them in water bath at $40{ }^{\circ} \mathrm{C}$ for 30 minutes, and then place them in ice water bath for 5 minutes. Take the first group as the blank control group, measure the absorbance of each group at $490 \mathrm{~nm}$, and calculate the polysaccharide content in sample solution by standard curve ${ }^{[8]}$.

\subsubsection{Polysaccharide Yield}

According to the polysaccharide content of pitaya fruit, calculate the total amount of polysaccharide in the treated 
pitaya, and then divide the polysaccharide mass obtained by the total amount of polysaccharide, so as to obtain polysaccharide yield.

Polysaccharide yield $(\%)=$ polysaccharide mass $/$ pitaya powder mass $\times 100 \%$

\section{Results and Analysis}

\subsection{Single factor test}

The yield of polysaccharides is affected by a variety of factors. This experiment focuses on the impact of three factors on the yield of polysaccharides, namely the extraction temperature, solid-liquid ratio and extraction time.

\subsubsection{Influence of Extraction Temperature on the Yield of Polysaccharide}

Under the same conditions of one-hour extraction time, two extraction times and a solid-liquid ratio of 1:20, change extraction temperature, and study its impact on the yield of polysaccharide. The experimental results are shown in Figure 2.

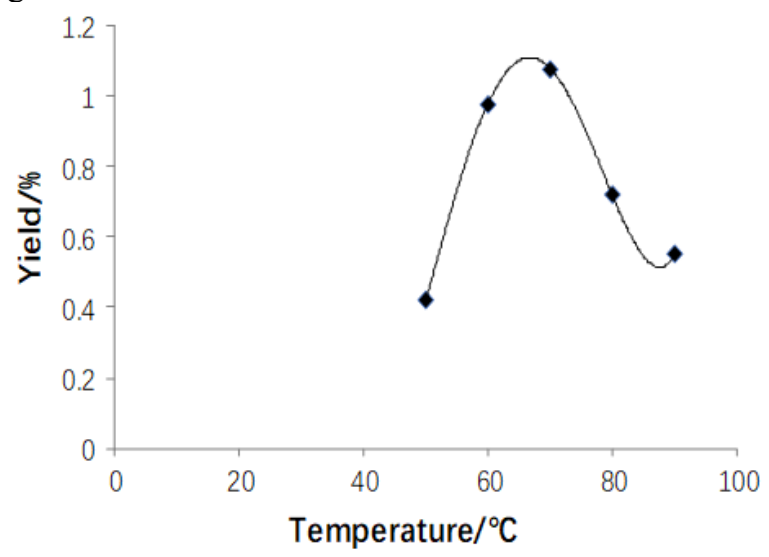

Fig. 2 Effect of extraction temperature on the yield of polysaccharide

As can be seen from Figure 2, under $70^{\circ} \mathrm{C}$, the yield of polysaccharides gradually increased with the increase of extraction temperature. Above $70{ }^{\circ} \mathrm{C}$, the yield of polysaccharides decreased with the increase of extraction temperature.

The analytical reason is that the yield of polysaccharides decreases when the temperature is too high. It is likely that the temperature is too high, so the polysaccharides are destroyed, the glycosidized bond is broken, and the polysaccharide degrades continuously, which finally leads to the decrease of the yield of polysaccharides ${ }^{[9]}$. Therefore, the appropriate temperature is $70^{\circ} \mathrm{C}$.

\subsubsection{Effect of Solid-liquid Ratio on the Yield of Polysaccharide}

Under the same conditions of extraction temperature $70^{\circ} \mathrm{C}$, one-hour extraction time and two extraction times, change solid-liquid ratio, study its impact on the yield of polysaccharide. The experimental results are shown in Figure 3.

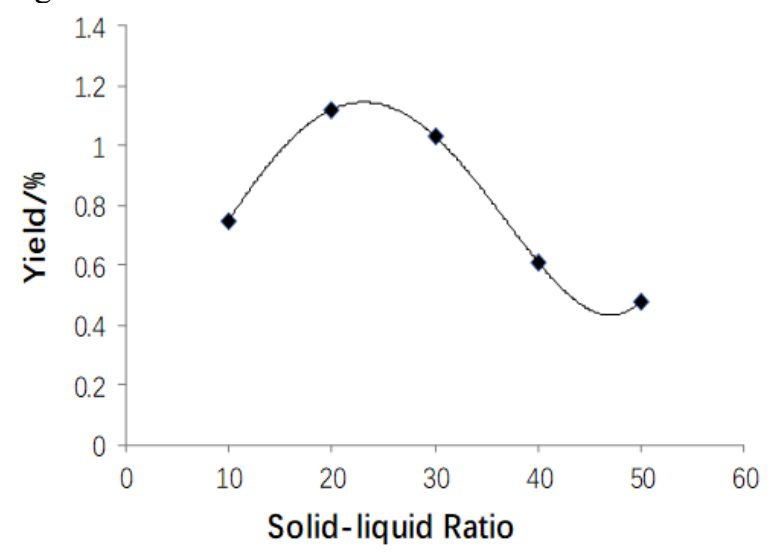

Fig. 3 Effect of solid-liquid on the yield of polysaccharide

As can be seen from Figure 3, the yield of polysaccharide increased with the increase of the solidliquid ratio when it was under 1:20, and after the solidliquid ratio increased above 1:20, the yield of polysaccharide was inversely proportional to the solidliquid ratio. The analytical reason is as follows. When the solid-liquid ratio is above $1: 20$, with the increase of solidliquid ratio, more and more distilled water is used for extraction, which increases the consumption of subsequent processes, and the loss of polysaccharides is also increasing ${ }^{[10]}$. Therefore, in order to ensure a good polysaccharide yield, the solid-liquid ratio of 1:20 is the most appropriate choice in the experiment.

\subsubsection{Influence of Extraction Time on the Yield of Polysaccharides}

Under the same conditions of extraction temperature $70^{\circ} \mathrm{C}$, a solid-liquid ratio of 1:20 and two extraction times, change extraction time, study its effects on the yield of polysaccharide. The experimental results are shown in Figure 4.

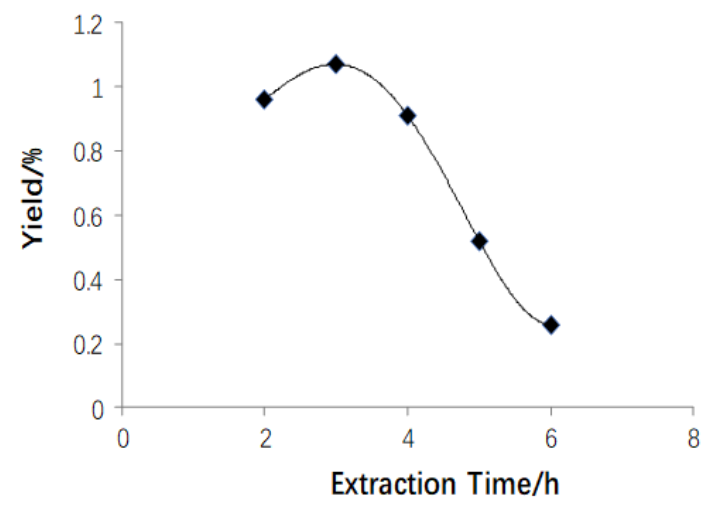

Fig. 4 Influence of extraction time on the yield of polysaccharide

According to the change rule in Figure 4, the yield of polysaccharides increased with the extension of extraction time. However, after 3 hours, the yield of polysaccharides decreased significantly with the increase of extraction time. The reason is as follows. As the extraction is done in hot water, the effect of too long extraction time is similar to that of too high temperature, which will lead to the breaking of the glycosidized bond 
and the continuous degradation of polysaccharides. Therefore, too long extraction time will lead to a decrease in the yield of polysaccharides ${ }^{[11]}$. Therefore, 3 hours is the most appropriate extraction time.

\subsection{Orthogonal Test}

Orthogonal test is a design method to study multi-factors and multi-levels. On the basis of single factor experiment, orthogonal test is carried out by selecting some representative points, and the horizontal combination selected by orthogonal test is listed into a table, which is called orthogonal table ${ }^{[12]}$.

According to the conditions obtained from the singlefactor test and considering various factors, this experiment took the extraction temperature, liquid-solid ratio and extraction time as factors, and $\mathrm{L}_{9}\left(3^{4}\right)$ orthogonal method was adopted to design the experiment, as shown in Table 2.

Table 2 Factor level table

\begin{tabular}{cccc}
\hline & & Factors & \\
Levels & Extraction Temperature $/{ }^{\circ} \mathrm{C}$ & Extraction Time $/ \mathrm{h}$ & Solid-liquid Ratio \\
& $\mathrm{A}$ & $\mathrm{B}$ & $1: 10$ \\
\hline 1 & 60 & 2 & $1: 20$ \\
2 & 70 & 3 & $1: 30$ \\
\hline
\end{tabular}

Table 3 Orthogonal test

\begin{tabular}{ccccc}
\hline NO. & A & B & C & Yield $/ \%$ \\
\hline 1 & 60 & 2 & $1: 10$ & 0.84 \\
2 & 60 & 3 & $1: 30$ & 1.13 \\
3 & 60 & 4 & $1: 20$ & 0.43 \\
4 & 70 & 2 & $1: 20$ & 0.88 \\
5 & 70 & 3 & $1: 10$ & 0.97 \\
6 & 70 & 4 & $1: 30$ & 0.45 \\
7 & 80 & 2 & $1: 30$ & 0.79 \\
8 & 80 & 3 & $1: 20$ & 0.68 \\
9 & 80 & 4 & $1: 10$ & 0.52 \\
$\mathrm{~K}_{1}$ & 2.40 & 2.51 & 2.33 & \\
$\mathrm{~K}_{2}$ & 2.30 & 2.78 & 1.99 & \\
$\mathrm{~K}_{3}$ & 1.99 & 1.40 & 2.37 & 0.38 \\
$\mathrm{R}$ & 0.41 & 1.38 & & \\
\hline
\end{tabular}

According to the range analysis in the table above, it can be seen that the degree of influence of various factors on the experimental results is $\mathrm{B}>\mathrm{A}>\mathrm{C}$, namely extraction time $>$ extraction temperature $>$ solid-liquid ratio. According to the experimental results, the optimal extraction conditions are as follows. The extraction time is maintained at $3 \mathrm{~h}$, the extraction temperature is $60^{\circ} \mathrm{C}$, and the solidliquid ratio is $1: 30 \mathrm{~g} / \mathrm{mL}$, under which the yield of polysaccharide is $1.13 \%$.

Table 4 Analysis of Variance Table

\begin{tabular}{ccccc}
\hline Factors & SS & Freedom & MS & F \\
\hline Extraction & 0.0305 & 2 & 0.01525 & 0.6531 \\
Temperature(A) & & & & \\
Extraction & 0.3556 & 2 & 0.17830 & 7.6360 \\
$\quad$ Time(B) & & & & \\
$\begin{array}{c}\text { Solid-liquid } \\
\text { Ratio (C) }\end{array}$ & 0.0291 & 2 & 0.01455 & 0.6231 \\
$\quad$ Error & 0.0467 & 2 & 0.02335 & \\
\hline
\end{tabular}

Look up the $\mathrm{F}$ distribution threshold table, we can get $\mathrm{F}_{0.10(2,2)}=9.00, \mathrm{~F}_{0.05(2,2)}=19.00$. According to the table above, the value of $F$ is small, which can be concluded that the three factors have no significant influence on the yield of polysaccharides. The reason may be that the experimental error e in this experiment is large, and the error degree of freedom is small, which is only 2, leading to low sensitivity of the experiment ${ }^{[13]}$. Since the influence of each factor is not significant, there is no need to carry out multiple comparisons between each factor level. Therefore, the result obtained from range analysis in Table 3 is the optimal level combination [14]

\section{Conclusion}

This paper used the ethanol subsiding method to extract polysaccharide from pitaya and investigated the effects of extraction temperature, solid-liquid ratio, extraction time and other factors on the extraction yield of polysaccharides. The optimum extraction technology of pitaya was determined by orthogonal test. The optimized conditions are as follows. The sample is added into water according to the solid-liquid ratio of 1:30, the extraction temperature is kept at $60^{\circ} \mathrm{C}$, and the extraction time is $3 \mathrm{~h}$. Under the optimized conditions, the yield of pitaya polysaccharide was $1.13 \%$. This experiment provides a 
theoretical basis for industrial large-scale extraction of pitaya polysaccharide.

\section{Fund program}

Natural Science Research Foundation of the Department of Education of Anhui Province (No. KJ2020A0789); Anhui provincial-level quality engineering project: (2020jyxm0790, 2020zyrc077, 2020zyrc081, 2019jxjj45); school-level grassroots teaching and research office demonstration project of Anhui Xinhua University (2019jyssfx02); Research project of Anhui Xinhua University (kytd201908).

\section{References}

1. He CF, Li P, Zhao J, etc. Extraction and structure identification of polysaccharide from pitaya stem $[\mathrm{J}]$. Food and Fermentation Industries, 2009, 11:140-143.

2. Zhou LP, Guo XH. Bioactive components from the stems of pitaya and their development and application prospects [J]. Food Research and Development, 2007, 28(7):169-172.

3. Zhang P, He MP, Yin L, etc. Study on protein removal process of pomegranate peel polysaccharide by Sevage method $[\mathrm{J}]$. Food Science and Technology, 2013,12:219-222+231.

4. Gao HY, Wang Q, Yu YB. Research status and development prospect of pitaya flower [J]. Journal of Tropical Biology, 2012,3(3):281-286.

5. Gao HY, Wang Q, Chen Y, etc. Optimization of extraction process of polysaccharide from pitaya flower by water [J]. Fujian Journal of Agricultural Sciences, 2014,28(4):335-338.

6. Xue D, Huang DD, Huang GH, etc. Research progress on extraction, isolation and purification of plant polysaccharides $[\mathrm{J}]$. Journal of Chinese Medicinal Materials, 2014, 37(1):157-161.

7. Liu J, Wang W, etc. Study on extraction and antioxidant activity of papaya polysaccharide [J]. Journal of Henan University of Technology, 2011, 32(1): 48-52.

8. Wu GF, Tang J. Purification and vitro antioxidant activity of aloe polysaccharide [J]. Journal of Henan University of Technology, 2002,23(2):75-78.

9. Zhang Q, Zhang TM. Determination of polysaccharide content by phenol-sulfuric acid colorimetry $[\mathrm{J}]$. Food and Drug, 2004, (7):17-18.

10. Sun F, Gu WY, etc. Extraction technology of crude polysaccharide from yam [J]. Journal of Food Science and Biotechnology, 2006, 25 (3): 79-83.

11. Cai JY, Xu JR, Sun S, etc. Optimization of microwave extraction of polysaccharide from pitaya fruit by response surface methodology [J]. Journal of Henan University of Technology (Natural Science Edition), 2015,03:65-70.

12. Gao HY, Wang Q, Chen Y, etc. Study on microwaveassisted extraction of polysaccharide from pitaya flower [J]. Fujian Journal of Agricultural Sciences, 2014,29(9):909-912.

13. Feng YX. Biological characteristics, cultivation techniques and development prospects of pitaya fruit [J]. Journal of Yunnan Tropical Crops Science \& Technology, 2001,25(3):36-40.

14. Song LH, Xiao W, etc. The extraction technology of ginseng polysaccharide optimized by orthogonal test [J]. Chinese Traditional and Herbal Drugs, 2012,43 (2): 283-287. 